\title{
Fission Neutron Tomography of a 280-L Waste Package
}

\author{
T. Bücherl ${ }^{*}$, Ch. Lierse von Gostomski, T. Baldauf
}

ZTWB Radiochemie München, Technische Universität München, Walther-Meißner-Str. 3, 85748 Garching, Germany

*thomas.buecherl@tum.de

Keywords: Fission Neutron, Transmission Tomography, Emission Tomography, NECTAR, Radioactive Waste, Non-Destructive Characterization

\begin{abstract}
Based on a recent feasibility study, where it is demonstrated that fission neutron radiography of 200-l (radioactive) waste drums is possible at NECTAR, these investigations are extended to fission neutron tomography in transmission and emission mode. As sample, a 280-1 drum is used containing inactive waste being typical for radioactive waste drums. For emission tomography, an AmBe-neutron source is measured separately and at different positions in the drum.
\end{abstract}

\section{Introduction}

For the non-destructive characterization of radioactive waste packages for the declaration or verification of their radioactive inventory, well-established passive and active methods are applied. These are mainly based on gamma-spectroscopic emission measurements (segmented gamma scanning), gamma-transmission measurements (e.g. radiography and tomography) using intense external radionuclide sources (e.g. ${ }^{60} \mathrm{Co}$ ) or accelerators, neutron emission counting with time correlation analysis to distinguish between neutrons originating from spontaneous fission and $(\alpha, n)$ events, and neutron interrogation techniques inducing fission events. Tomography using fission neutrons, in transmission and emission mode, is to the best of our knowledge not applied on waste packages, yet.

In a feasibility study[1] it was demonstrated that fission neutron radiography of 200-l (radioactive) waste drums is possible at NECTAR, a neutron radiography and tomography facility using fission neutrons [2]. In a subsequent step, the study was extended on tomographic investigations of 200-l and 280-l mock-up waste drums both in transmission and emission mode.

\section{Experimental}

NECTAR Facility. The measurements are performed at the NECTAR facility at the research reactor FRM II of the Technical University Munich (TUM) using an ANDOR iKon L 4 Megapixel back illuminated CCD camera operating at $-100{ }^{\circ} \mathrm{C}$ with a Nikkor $100 \mathrm{~mm} \mathrm{f} / 2.8$ lens, resulting in a field of view of $291 \mathrm{~mm} \times 288 \mathrm{~mm}$ (width $\mathrm{x}$ height). The conversion of fission neutrons to blue light $(450 \mathrm{~nm})$ is performed by a PP/ZnS:Ag (30\%) scintillator with dimensions $2.4 \mathrm{~mm}$ x $400 \mathrm{~mm}$ x 400 mm (depth x width x height) distributed by RC TRITEC, Switzerland, attached to the light tight housing of the detector box by a sliding mechanism. The L/D-value used for all transmission measurements is $132 \pm 12$ [3].

Sample. The sample is a 280-l drum with $100 \mathrm{~cm}$ in height, $71 \mathrm{~cm}$ in diameter and a mass of about $500 \mathrm{~kg}$. It houses a 200-l drum containing two supercompacted pellets on the bottom and then filled up with metallic and plastic scrap (Fig. 1). A cylindrical container made of polyethylene (PE) is used for housing additional neutron sources. 

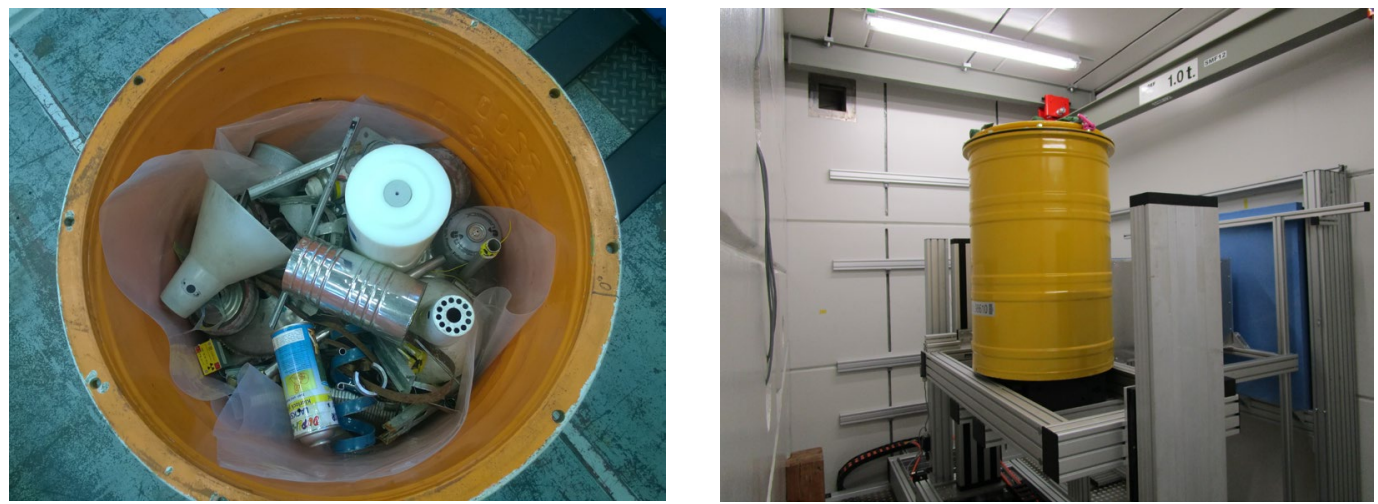

Fig. 1: Left: Open 200-l drum during assembling with two pellets covered by scrap. The white PE-cylinder can ingest neutron sources. Right: 280-l drum on the sample manipulator of NECTAR.

External Neutron Sources. For the feasibility tests of neutron emission tomography, two certified AmBe-sources with neutron emission rates of $2.2 \cdot 10^{5} \mathrm{~s}^{-1}$ and $2.2 \cdot 10^{6} \mathrm{~s}^{-1}$ are available. Their mean neutron energy is about $2.7 \mathrm{MeV}$ with a significant amount (about 23\%) of neutrons below $1 \mathrm{MeV}$ having a mean energy of $400 \mathrm{keV}$. The compacted mixture of $\mathrm{AmO}_{2^{-}}$and Beryllium-metal-powder is housed in a sealed container of $6 \mathrm{~cm}$ height and $3 \mathrm{~cm}$ diameter.

Measurements. The first series is focused on transmission measurements. The 280-l drum is positioned as close as possible to the detector still enabling undisturbed rotation, i.e. at a distance of $38 \mathrm{~cm}$ between the axis of rotation and the scintillator screen. As the FOV of the detector system is smaller than the diameter of the drum, the sample is translationally scanned, i.e. one projection is setup by three transmission images differing in position by $23 \mathrm{~cm}$ each. This results in an overlap of successive images of $6.1 \mathrm{~cm}$. The integration time for each image is $60 \mathrm{~s}$. In total 491 angular projections for $360^{\circ}$ are measured applying the golden ratio method [4]. To minimize the deadtime of the system due to mechanical movements, the translational scanning is performed by reciprocating motion. The total measuring time is about 1.5 days, Dark images and open beam images are measured additionally.

In the second series, four runs in total are investigating the feasibility of the NECTAR facility for emission tomography. The first run is performed without drum, positioning the strong AmBesource in the axis of rotation of the turntable at a distance of $3 \mathrm{~cm}$ to the center of the fission neutron scintillator screen. The measurement is using the golden ratio method with 207 projections and an integration time of $3500 \mathrm{~s}$ for each projection, a total measuring time of almost 9 days. The next runs are performed with drum, positioned identically like in the transmission measurement. First, the weak AmBe-neutron source is ingested in the PE-container, then it is replaced by the strong AmBe-neutron source in the second run. In the last run this source is positioned close to the outer side of the PE-container avoiding moderation by the PE. The parameters for the last three runs are the same like for the transmission measurements, except the fission neutron beam of the reactor is closed. Additional test measurements with different scintillators being sensitive to thermal neutrons are performed, too.

\section{Data Evaluation}

Preprocessing. All images are filtered [5] to remove artefacts caused by gamma-radiation and/or scattered neutrons hitting the CCD sensor directly, followed by dark-image correction and normalization [6]. 
The stitching of images to get a projection is performed by using a minimization routine, i.e. by superimposing the left and right parts of two images, estimating the minimum of the sums of the absolute differences of all pixels as a function of the width of superimposition. As the intensity of the neutron beam is differing between the right and left side of each image and thus the uncertainties of each pixel value of the normalized image, an appropriate weighing matrix is taken into account. Fig. 2 shows as an example three normalized images for one projection and the resulting stitched projection.
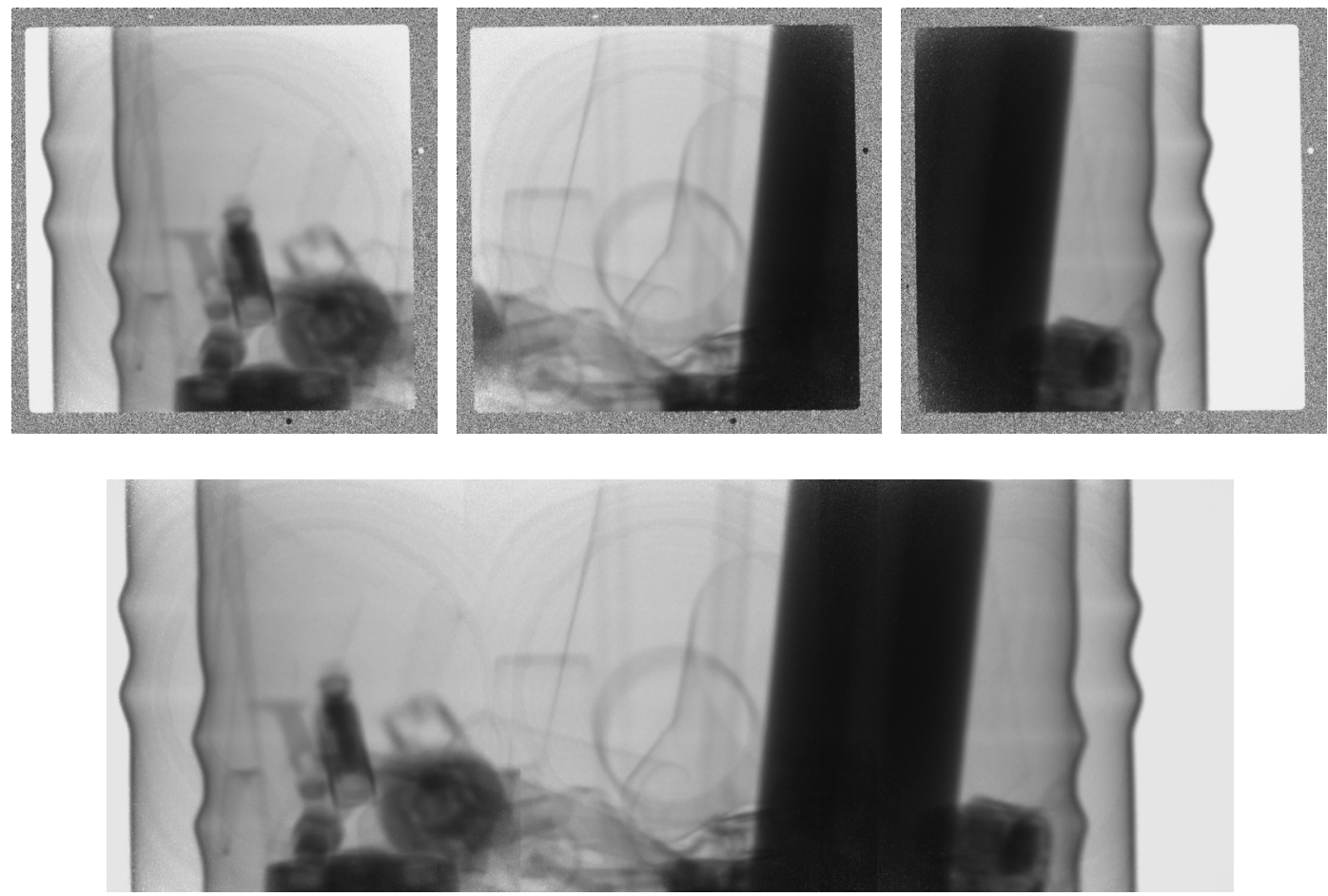

Fig. 2: Upper row: Three normalized radiographs making up one projection. Note, that the images are not cropped, i.e. the area not covered by the scintillator but seen by the CCD is visible. Bottom: Stitched projection estimated from the cropped images in the upper row.

Reconstruction Algorithms. For reconstruction of the transmission data, the filtered backprojection (FBP) method provided by the program Octopus [7] is applied, assuming parallel beam geometry. The emission data are reconstructed applying the FBP algorithm with rampfilter and the maximum likelihood expectation maximization (MLEM) [8], using python scripts. Here, the projection data is binned by five pixels to minimize computation time for this feasibility study.

\section{Results and Discussion}

Transmission Measurements. The reconstruction of the transmission data does not result in utilizable data, as the tomograms are blurred and no details can be identified (Fig. 3). This is based on two effects, the low dynamic range of the data of 660 grey values, and more problematic, the effect of beam divergence. The latter is demonstrated by comparing the stitching parameters of projections at $52.52^{\circ}$ and $327.54^{\circ}$ with an estimated overlap of two images of 189 and 143 pixels, respectively (Fig. 4). For the assumed parallel beam geometry, the value of overlap must be constant, while indicating a change of 46 pixels, i.e. a shift of about 7 
$\mathrm{mm}$, due to a maximum beam divergence of $2.1^{\circ}$ [9]. Thus, an improved iterative method for a combined stitching and reconstruction process has to be developed, taking into account beam divergence and changing uncertainties in the pixel values due to non-homogeneous areal beam intensity distribution.
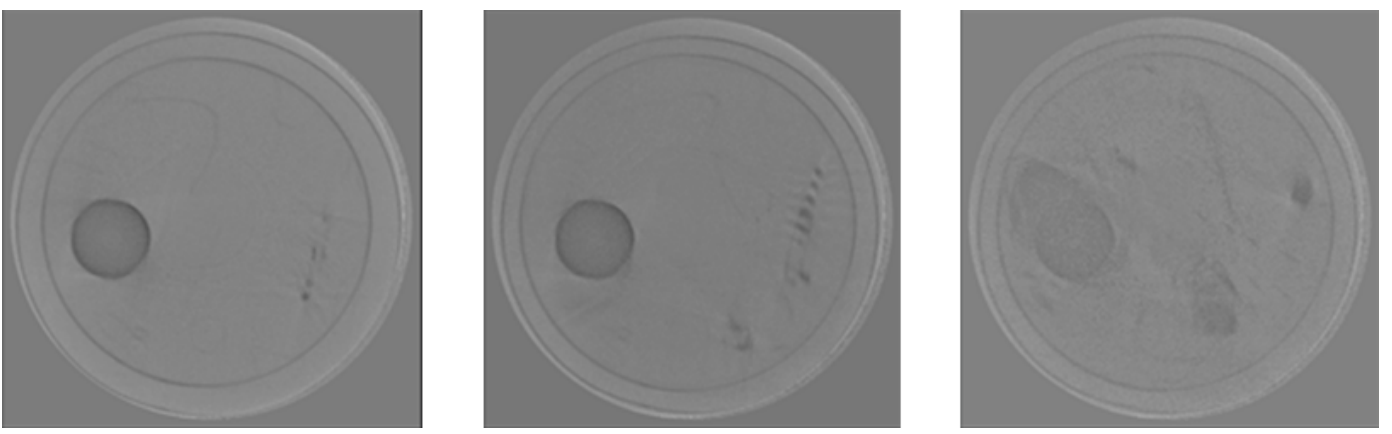

Fig. 3: Three tomograms at different height positions of the drum. The borders of the 280-l-and 200-l-drums are visible as well as the PE-container, but no further details.
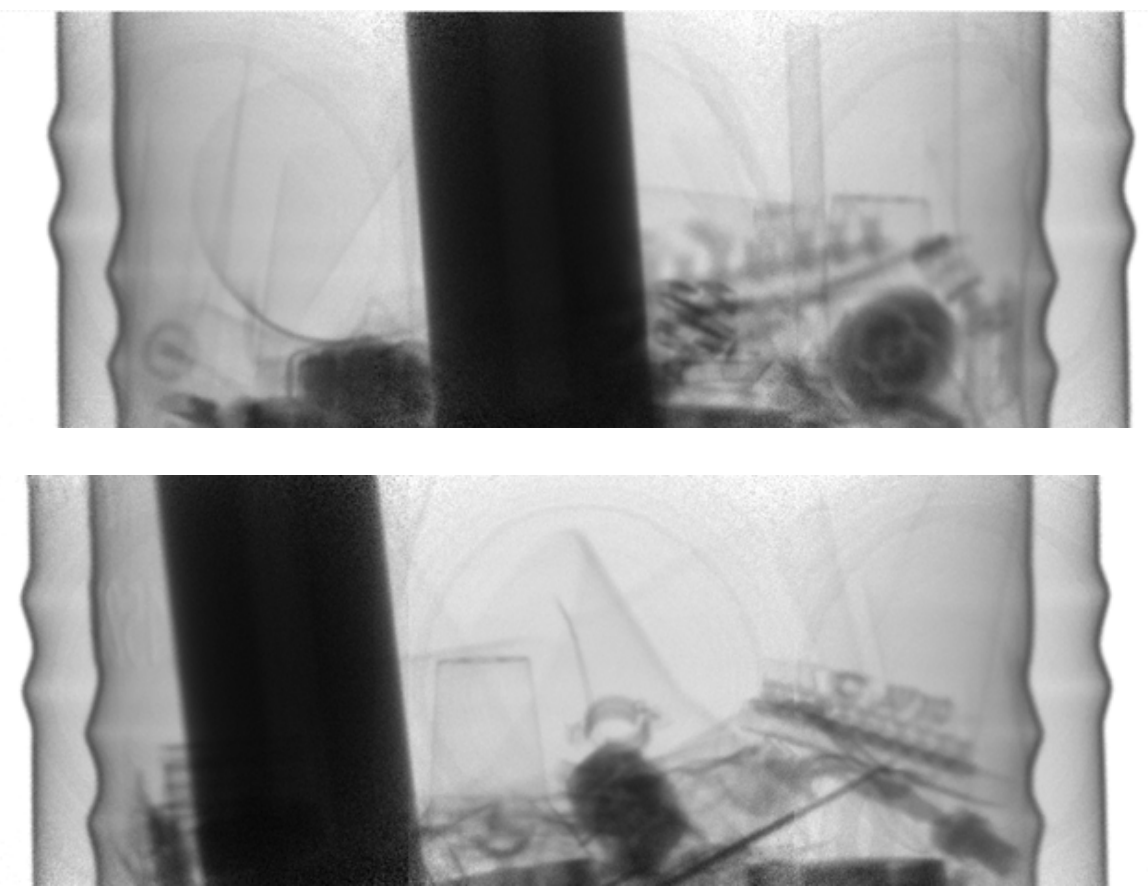

Fig. 4: Projection at $52.52^{\circ}$ (top) and $327.54^{\circ}$ (bottom) with an overlap of two adjacent images of 189 and 143 pixels, respectively. A shift of the axis of rotation is present, too.

A first idea of such an algorithm is sketched in Fig. 5. For the presented measurement, three identical neutron sources at distances $d$ being the translational shift of the manipulator of successive image positions contribute to one projection, taking into account the beam divergences and the overlap. Using an ART algorithm modified for the use of multiple external sources, an improved image quality may be achieved. This is the work of a future R\&D project. 


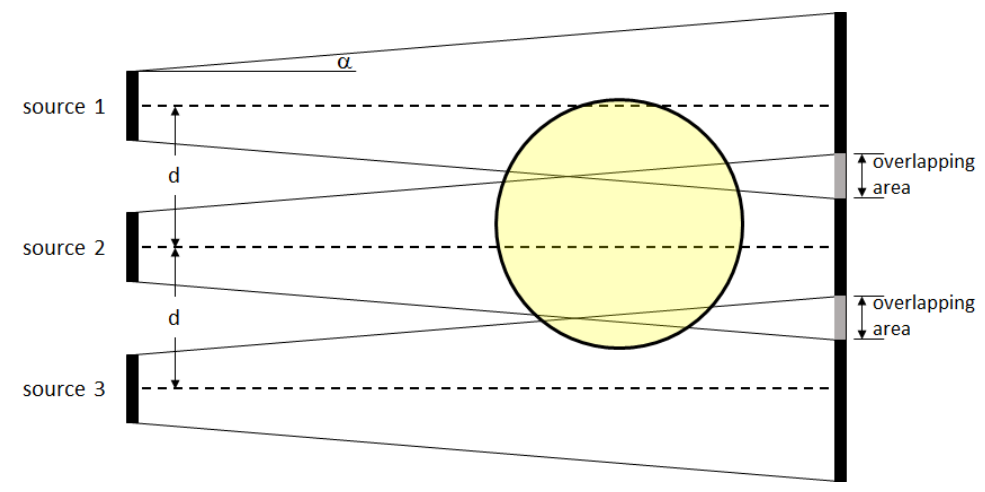

Fig. 5: Sketch of the basic idea for an improved combined stitching and reconstruction algorithm taking into account a beam divergence $\alpha$.

Emission Measurements. Fig. 6 shows the neutron activity distributions of the first run using the strong AmBe-source evaluated by MLEM and FBP. For data evaluation a binning of 8x8 pixels is applied. The horizontal line profiles through the center of the distributions indicate that the influences of scattering and absorption in the sample are not taken into account completely and an improved data evaluation has to be performed.

The images of the three other emission measurement runs with the samples in the drum show no significant signals in the detector for all scintillators used, indicating that the detection efficiency at NECTAR is too low. A rough calculation for the AmBe-source emitting $2.2 \cdot 10^{6} \mathrm{~s}^{-1}$ neutrons positioned in the center of a 200-l barrel, i.e. at $30 \mathrm{~cm}$ distance from the detector plane, results in about $900 \mathrm{~cm}^{-2} \mathrm{~s}^{-1}$ or 0.02 pixel $^{-1} \mathrm{~s}^{-1}$ neutrons in this plane. These values do not consider attenuation and moderation effects. In combination with the actual detection efficiency for fission neutrons of less than $1 \%$, their detection is unlikely within reasonable integration times. Thus an improvement in detection efficiency is one of the most important topics for transmission and emission measurements.
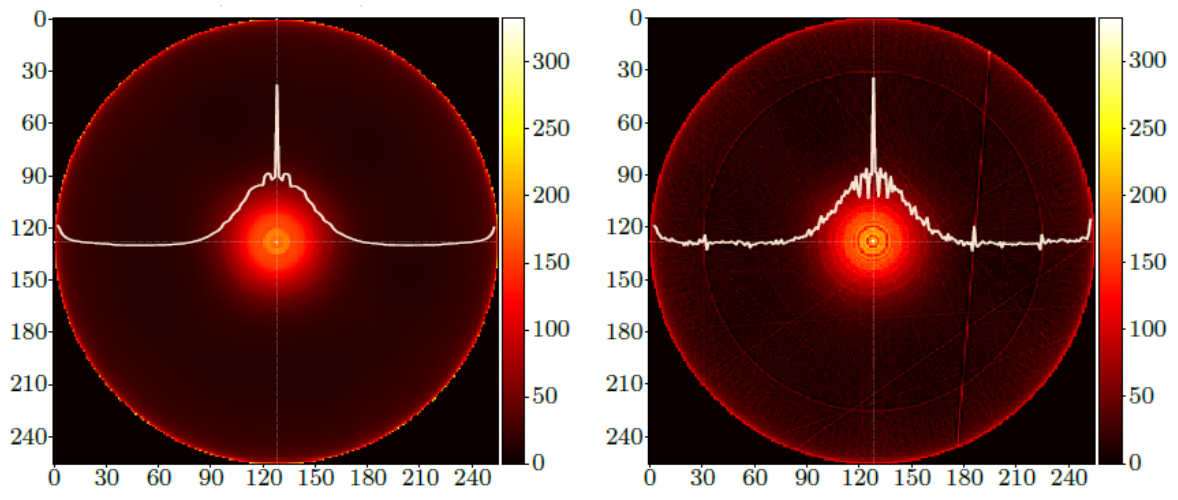

Fig. 6: MLEM-reconstruction after 30 iterations and smoothing filter applied (left) and FBP (right). The axes are given in pixels (i.e. $256 \times 256$ pixels), with one pixel corresponding to about $0.78 \mathrm{~mm}$ in size. The line profiles show the horizontal distributions of the neutron activity.

\section{Summary}

The results of the study show, that transmission tomography using fission neutrons on large sized samples like 280-1 drums is feasible, but requires further developments in a combined stitching 
and reconstruction algorithm as well as for increased detection efficiency. A possible toehold for an algorithm is sketched. The applicability will strongly depend on density, thickness and distribution of the different materials in the drums. It is demonstrated that neutron emission tomography is possible at the NECTAR facility, although only applicable in praxis on large samples for extremely high neutron emission rates. Here passive neutron assay systems like SANDRA [10] are still in favor due to their much higher detection efficiency, but not giving the activity distribution in the volume of the sample. Developments for improved detection efficiencies for fission neutrons can overcome these limitations.

\section{References}

[1] T. Bücherl, O. Kalthoff, Ch. Lierse von Gostomski, A feasibility study on reactor based fission neutron radiography of 200-l waste packages, Physics Procedia 88 (2017) 64 - 72. https://doi.org/10.1016/j.phpro.2017.06.008

[2] NECTAR: Heinz Maier-Leibnitz Zentrum. (2015). NECTAR: Radiography and tomography station using fission neutrons. Journal of large-scale research facilities, 1, A19. https://doi.org/10.17815/jlsrf-1-45

[3] T. Bücherl, Ch. Lierse von Gostomski, H. Breitkreuz, M. Jungwirth, F.M. Wagner, NECTAR - A fission neutron radiography and tomography facility, Nucl. Instr. Meth. Phys. Res. A 651(2011) 86-89. https://doi.org/10.1016/j.nima.2011.01.058

[4] L. G. Butler, E. H. Lehmann, B. Schillinger, Neutron Radiography, Tomography, and Diffraction of Commercial Lithium-ion Polymer Batteries, Physics Procedia, 43, 2013, 331-336, https://doi.org/10.1016/j.phpro.2013.03.039.

[5] K. Osterloh, T. Bücherl, Ch. Lierse von Gostomski, U. Zscherpel, U. Ewert, S. Bock, Filtering algorithm for dotted interferences, Nucl. Instr. Meth. Phys. Res. A 651(2011) 171-174. https://doi.org/10.1016/j.nima.2011.01.107

[6] A. C. Kak, M. Slaney, Principles of Computerized Tomographic Imagimg, IEEE Press, 1988, ISBN 0-87942-198-3.

[7] M. Dierick, B. Masschaele, L, Van Hoorebeke, Octopus, a fast and user-friendly tomographic reconstruction package developed in LabView, Measurement Science and Technology, Volume 15, Number 7, 2004. https://doi.org/10.1088/0957-0233/15/7/020

[8] http://campar.in.tum.de/twiki/pub/Main/MoritzBlume/EMPET.pdf (last call 07.11.2018).

[9] J. Guo, T. Bücherl, Y. Zou, Z. Guo, Study on beam geometry and image reconstruction algorithm in fast neutron computerized tomography at NECTAR facility, Nucl. Instr. Meth. Phys. Res. A, 651 (2011) 180-186. https://doi.org/10.1016/j.nima.2011.01.097

[10] http://www.en-trap.eu/doc/neutronsynopsis.pdf (last call 07.11.2018). 